\title{
Comment to: Laparoscopic versus hybrid approach for treatment of incisional ventral hernia: a prospective randomized multicenter study of 1-month follow-up results. Ahonen-Siirtola, M., Nevala, T., Vironen, J. et al.
}

\author{
J. Li ${ }^{1} \mathbb{D}$
}

Received: 23 June 2018 / Accepted: 17 August 2018 / Published online: 22 August 2018

(c) Springer-Verlag France SAS, part of Springer Nature 2018

\section{Dear Editors:}

We read with great interest the recent article by AhonenSiirtola [1], published in Hernia. The authors reported that incisional hernia repair performed with hybrid method, including hernia sack resection combined with fascial closure, was associated with a low risk of enterotomy and diminished seroma formation compared to laparoscopic repair alone. We applaud the authors for the first multicenter randomized controlled trial and agree with the authors' interpretations on this issue, as well as the advantages provided by hybrid technique. Furthermore, we have a few comments on this topic. The first comment is on defect closure: the incisional hernia size in the present trial ranged from 2-7 cm, which is relative small or middle size, and actually, this sized hernia defect could be closed laparoscopically without difficulty; one suggested modality is the use of barbed suture, which had greatly facilitated defect closure, as we did in laparoscopic direct hernia defect closure, in which the hernia size was over 3-4 cm [2]. My second comment is about the necessity of sac resection during incisional hernia repair: still there is no direct evidence to support the advantage of sac resection, and in our opinion, hernia sac closure itself could be sufficient enough to prevent postoperative bulge and seroma formation, especially in small- to medium-sized hernias which could be performed by laparoscopic technique. By defect closure, sac peritoneum contact, approximation, and eradication of the defect cavity, the

This comment refers to the article available online at https://doi. org/10.1007/s10029-018-1784-2.

$\triangle \mathrm{J} . \mathrm{Li}$

Lijunshenghd@126.com

1 Department of General Surgery, Affiliated Zhongda Hospital, Southeast University, Nanjing 210009, China seroma formation and bulge could be prevented. In addition, we agree with the author and we want to emphasize that the criteria for hybrid technique should be liberal; if you think hybrid technique is needed due to heavy adhesion or dubious intestine lesion, no matter how fleeting that thought, a hybrid technique should be taken since the consequence of intestine leakage is a disaster.

Again, we congratulate Ahonen-Siirtola et al. for their multicenter work and we hope that their contribution will be useful to other surgeons who are treating incisional hernias.

Funding None.

\section{Compliance with ethical standards}

Conflict of interest No conflict of interest to be declared by the authors.

Ethical approval This article did not require ethical approval of any kind.

Human and animal rights This article does not contain any studies with human participants or animals performed by any of the authors.

Informed consent This article does not include patients and, therefore, informed consent was not applicable.

\section{References}

1. Ahonen-Siirtola M, Nevala T, Vironen J, Kössi J, Pinta T, Niemeläinen S, Keränen U, Ward J, Vento P, Karvonen J, Ohtonen P, Mäkelä J, Rautio T (2018) Laparoscopic versus hybrid approach for treatment of incisional ventral hernia: a prospective randomized multicenter study of 1-month follow-up results. Hernia. https://doi.org/10.1007/s10029-018-1784-2 (Epub ahead of print)

2. Li J, Zhang W (2018) Closure of a direct inguinal hernia defect in laparoscopic repair with barbed suture: a simple method to prevent seroma formation? Surg Endosc 32(2):1082-1086 